\title{
Control of spatially patterned synchrony with multisite delayed feedback
}

\author{
C. Hauptmann, ${ }^{1,2}$ O. Omel 'chenko, ${ }^{1,2,3}$ O. V. Popovych, ${ }^{1,2}$ Y. Maistrenko, ${ }^{1,2,3}$ and P. A. Tass ${ }^{1,2,4}$ \\ ${ }^{1}$ Institute of Neuroscience and Biophysic -3, Research Center Jülich, D-52425 Jülich, Germany \\ ${ }^{2}$ Virtual Institute of Neuromodulation, Research Center Jülich, D-52425 Jülich, Germany \\ ${ }^{3}$ Institute of Mathematics, National Academy of Sciences of Ukraine, 01601 Kyiv, Ukraine \\ ${ }^{4}$ Department of Stereotaxic and Functional Neurosurgery, University Hospital, 50924 Cologne, Germany \\ and Brain Imaging Center West, D-52425 Jülich, Germany
}

(Received 8 December 2006; revised manuscript received 10 May 2007; published 20 December 2007)

\begin{abstract}
We present an analytical study describing a method for the control of spatiotemporal patterns of synchrony in networks of coupled oscillators. Delayed feedback applied through a small number of electrodes effectively induces spatiotemporal dynamics at minimal stimulation intensities. Different arrangements of the delays cause different spatial patterns of synchrony, comparable to central pattern generators (CPGs), i.e., interacting clusters of oscillatory neurons producing patterned output, e.g., for motor control. Multisite delayed feedback stimulation might be used to restore CPG activity in patients with incomplete spinal cord injury or gait ignition disorders.
\end{abstract}

DOI: 10.1103/PhysRevE.76.066209

PACS number(s): 05.45.Xt, 87.19.La

\section{INTRODUCTION}

Spatially homogenous as well as spatially patterned synchronization processes abound in physical, chemical, and biological systems $[1,2]$. Several neurological diseases are characterized by pathologically strong synchronization of neuronal activity. To improve current therapies [3], recent studies have focused on the development of control techniques which counteract synchronization processes by desynchronization, utilizing phase resetting [4] or delayed feedback [5-7] principles (see [8]).

Another fundamental challenge is the development of techniques for the control of spatially patterned synchronization processes. These techniques are relevant, e.g., in the context of central pattern generators (CPGs), which are neural networks that can endogenously (i.e., without external drive) produce oscillatory patterned outputs [9-13]. CPGs control a variety of functions, e.g., ongoing oscillatory movements such as breathing, chewing, walking, running, swimming, and flying, in both animals and/or humans [9-13]. CPGs are located in the spinal cord, the brain stem, and are even considered to be fundamental functional units of the neocortex [14]. Typically, CPGs consist of different ensembles of synchronously active neurons, where the different ensembles act in a well-coordinated manner [9-12], e.g., phase-locked to each other, in order to control the sequential action of different limbs during locomotion [14]. Modeling approaches have shown their relevance for the investigation of the role of CPGs for the control of limb locomotion [15].

Dysfunctions of CPGs cause a variety of severe disorders $[10-13,16,17]$. For instance, in patients suffering from incomplete spinal cord injury, treadmill training is performed to reestablish CPG action and hence improve locomotion $[10,17]$. To further advance such therapies, it might be promising to develop electrical stimulation techniques which specifically restore CPG activity by a direct control of the CPG network [18]. With this aim in view, we present a control approach which enables the reliable induction of spatially patterned synchronization processes in a network of coupled oscillators, where in the absence of control the whole network may either be synchronized or incoherent, depending on the coupling strength chosen. Note that we do not assume any kind of preexisting spatial coupling pattern that might facilitate the emergence of the desired spatial patterns of synchrony. Rather, in our model the oscillators are globally coupled, which provides a nontrivial test scenario. To solve this problem, we use multisite delayed feedback stimulation. In previous studies, delayed feedback was used to control the state of a single oscillator [19] or to up or down regulate spatially homogenous synchronization $[5,6,20,21]$. By contrast, to evoke well-defined standing-wave or rotating-wavelike spatiotemporal patterns of synchrony, we administer linear delayed feedback at different sites of the network with different delays, respectively. Depending on the degree of overlap of the stimulation profiles of the different sites, we obtain different types of CPG-like activity. Intriguingly, our technique works in a demand-controlled way, so that minimal stimulation intensities are required to obtain the desired pattern of synchrony. We study our method in a network of coupled limit-cycle oscillators and propose possible medical applications.

\section{MODEL}

We start with an ensemble of globally coupled LandauStuart oscillators, representing a normal form of a supercritical Andronov-Hopf bifurcation, exposed to four-site delayed feedback stimulation according to

$$
\dot{W}_{j}=\left(1+i \omega_{j}-\left|W_{j}\right|^{2}\right) W_{j}+C\left(\bar{W}-W_{j}\right)+S_{j}(t),
$$

$j=1, \ldots, N$, where $\omega_{j}$ is the natural frequency of the $j$ th oscillator, $C$ is the global coupling strength, $\bar{W}=N^{-1} \sum_{k=1}^{N} W_{k}$ is the ensemble's mean field, and $N$ is the total number of oscillators. The mean field is delivered via four different electrodes $E_{1}, \ldots, E_{4}$ with four different delays $\tau_{1}, \ldots, \tau_{4}$ according to $S_{j}(t)=K \Sigma_{m=1}^{4} p_{m} \rho_{j m} \bar{W}\left(t-\tau_{m}\right)$, where the constant $K$ 
defines the strength of the four-site delayed feedback. In a practical application the local field potential might be the base for the delayed feedback stimulation signals, either recorded at the side of stimulation or at a remote side. The oscillators are arranged in a two dimensional lattice within the unit circle $D$ (Fig. 1). The stimulation topology, i.e., the strength with which the $m$ th electrode affects the $j$ th oscillator, is given by $\rho_{j m}$. The coefficients $p_{m}= \pm 1$ stand for the polarities of the corresponding stimulation signals. The four stimulation electrodes $E_{1}, \ldots, E_{4}$ are equally spaced around the neuronal population (Fig. 1).

For weak coupling and control, the oscillators' amplitudes can be eliminated $\left(\left|W_{j}\right| \rightarrow 1\right)$ and we get $[1,22]$

$$
\begin{aligned}
\dot{\Psi}_{j}= & \omega_{j}-\frac{C}{N} \sum_{k=1}^{N} \sin \left(\Psi_{j}-\Psi_{k}\right) \\
& -\frac{K}{N} \sum_{m=1}^{4} p_{m} \rho_{j m} \sum_{k=1}^{N} \sin \left[\Psi_{j}-\Psi_{k}\left(t-\tau_{m}\right)\right] .
\end{aligned}
$$

For the numerical analysis of Eq. (2), we add Gaussian white noise $F_{j}(t)$ to the right hand side of Eq. (2), with $\left\langle F_{j}(t)\right\rangle=0$, $\left\langle F_{j}(t) F_{k}\left(t^{\prime}\right)\right\rangle=A \delta_{j k} \delta\left(t-t^{\prime}\right)$, and constant noise amplitude $A$, so that only stable solutions are reached. Each phase oscillator serves as a model neuron, which fires whenever its phase $\bmod 2 \pi$ equals $0[1,4,23]$. $\rho_{j m}$ denotes the stimulation topology which is either segmental (Sec. III A, i.e., $\rho_{j m}=1$ if neuron $j$ belongs to subdomain $D_{m}, \rho_{j m}=0$ else, $\left.m=1, \ldots, 4\right)$ or overlapping (Sec. III B, i.e., $\rho_{j m}=\left[l d \sqrt{1+4\left(\frac{d}{l}\right)^{2}}\right]^{-1}$, where $d$ denotes the distance between electrode $m$ and neuron $j$, and $l$ is the length of the contacts of a typical deep brain stimulation electrode).

Obviously, system (2) is difficult to analyze for an arbitrary choice of natural frequencies $\omega_{j}$. Hence to gain some insight we consider the case of identical oscillators, i.e., $\omega_{j}=\bar{\omega}$. Even this case turns out to be far from trivial. We utilize here an approach similar to that developed for the Kuramoto model in the thermodynamic limit $N \rightarrow \infty$ (see [2] and references therein) where we use the continuous space variable $x$ instead of the discrete index $j$ of the oscillators. More precisely, for a large number of identical oscillators, where their spatial distribution within the unit circle $D$ remains uniform, system (2) can be replaced with its continuous analog,

$$
\begin{aligned}
\frac{\partial \psi(x, t)}{\partial t}= & \bar{\omega}-\frac{C}{\mu} \int_{D} \sin [\psi(x, t)-\psi(\xi, t)] d \xi \\
& -\frac{K}{\mu} \sum_{m=1}^{4} p_{m} \rho_{m}(x) \int_{D} \sin \left[\psi(x, t)-\psi\left(\xi, t-\tau_{m}\right)\right] d \xi
\end{aligned}
$$

where $\mu$ is the measure of the circle $D$ (see Fig. 1 ), $\bar{\omega}$ is the uniform natural frequency, and $\rho_{1}(x), \ldots, \rho_{4}(x)$ are nonnegative functions describing the stimulation profiles, i.e., the strength of stimulation via electrodes $E_{1}, \ldots, E_{4}$ at position $x$ [the latter definition obviously implies that $\rho_{j m}=\rho_{m}\left(x_{j}\right)$, where $x_{j}$ denotes the position of the $j$ th oscilla- tor]. The unknown function $\psi(x, t)$ denotes the phase of an individual oscillator at position $x$ at time $t$. In analogy to Eq. (2) the term proportional to $C$ corresponds to the global coupling, whereas the four terms proportional to $K$ describe the four-site delayed feedback stimulation. Equation (3) provides us with an opportunity to derive explicit analytical results, which, as we demonstrate in Sec. III, are in a good agreement with the results of the numerical simulations of Eq. (2). Moreover, although formulas obtained from Eq. (3) are developed for the case of identical oscillators, they turn out to approximate the results of the numerical simulations of Eq. (2) with distributed eigenfrequencies, too.

\section{RESULTS}

For model Eq. (2) we consider two different setups:

(i) Segmental stimulation. The four different subdomains $D_{m}$ (Fig. 1) are exclusively stimulated by their corresponding electrode $E_{m}$ (i.e., $\rho_{j m}=1$ if neuron $j$ belongs to subdomain $D_{m}, \quad \rho_{j m}=0$ else) with delay $\tau_{m}$ and polarity $p_{m}$ $(m=1, \ldots, 4)$. Segmental stimulation mimics a separate stimulation of four anatomically distinct, but interacting, populations, forming one spinal CPG [9-12,14,24].

(ii) Overlapping stimulation. Each neuron is affected by an overlap of all stimulation profiles, weighted according to its position relative to the electrodes. Overlapping stimulation models CPG inducing stimulation in only one, homogeneously connected population.

\section{A. Segmental stimulation}

Without stimulation $(K=0)$ the ensemble is synchronized, which is caused by the internal coupling [1]. Depending on the delays and polarities chosen, we induce qualitatively different spatiotemporal dynamics. Sequentially distributed delays [25] cause a rotating wave-type four-cluster state [Fig. 2(a)], where the population splits into four sequentially firing clusters [raster plot in Fig. 2(d)]. Noise and distributed natural frequencies $\omega_{j}$ make the phase distribution within each cluster slightly smeared. With the order parameters $[1,26]$

$$
R_{n}(t) \exp \left[i \Theta_{n}(t)\right]=\frac{1}{N} \sum_{k=1}^{N} \exp \left[i n \Psi_{k}(t)\right]
$$

we detect synchronization patterns, where $0 \leqslant R_{n} \leqslant 1$ for all times $t(n=1,2, \ldots)$ [27]. Directly after the onset of the stimulation, $R_{1}, R_{2}$, and $R_{3}$ approach zero, while $R_{4} \approx 0.8$, which indicates a four-cluster state [Fig. 2(b) (left)]. In contrast, with a grouped arrangement of the delays [28] a standing-wave-type two-cluster state emerges $\left(R_{1} \approx 0\right.$, $R_{2} \approx 1$, Fig. 2, right). Note that in both cases in each network element the multisite delayed feedback stimulation $S_{j}$ [i.e., the term proportional to $K$ in Eqs. (2) and (3)] nearly vanishes as soon as the desired spatiotemporal dynamics is established [Fig. 2(c)].

To derive the delays and polarities used in Fig. 2 [25,28], we consider the continuous model (3). We assume that $\rho_{m}(x)=\chi\left(x, D_{m}\right)$, where $D_{m}$ is the corresponding subdomain of $D$ (Fig. 1) and $\chi\left(x, D_{m}\right)$ is the characteristic function for 


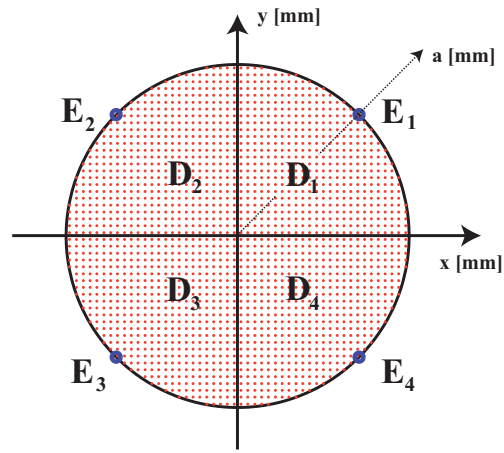

FIG. 1. (Color) Schematic figure of the network topology. The model neurons (red dots) are arranged within the unit circle and the four electrodes $E_{1}, \ldots, E_{4}$ (blue dots) are equally distributed on the diagonals at distance $a$ from the center of the network, with locations $X_{1}=(\widetilde{a}, \widetilde{a}), X_{2}=(\widetilde{a},-\widetilde{a}), X_{3}=(-\widetilde{a},-\widetilde{a})$, and $X_{4}=(-\widetilde{a}, \widetilde{a})$, with $\tilde{a}=a / \sqrt{2}$. The four different subdomains $D_{1}, \ldots, D_{4}$ are associated with the subpopulations mainly affected by the corresponding electrodes $E_{1}, \ldots, E_{4}$.

$D_{m}$, i.e., $\chi\left(x, D_{m}\right)=1$ if $x \in D_{m}$, and $\chi\left(x, D_{m}\right)=0$ if $x \notin D_{m}$. Then we rewrite Eq. (3) as

$$
\begin{aligned}
\frac{\partial \psi(x, t)}{\partial t}= & \bar{\omega}-\frac{C}{\mu} \int_{D} \sin [\psi(x, t)-\psi(\xi, t)] d \xi \\
& -\frac{K}{\mu} \int_{D} p_{m} \sin \left[\psi(x, t)-\psi\left(\xi, t-\tau_{m}\right)\right] d \xi
\end{aligned}
$$

We look for phase-locked solutions of Eq. (5) with a phase pattern characterized by four clusters and hence substitute the ansatz $\psi(x, t)=\Omega t+\phi_{m}\left(x \in D_{m}, m=1, \ldots, 4\right)$ into Eq. (5). In the simplest case of zero coupling all stable phase-locked solutions have frequencies $\Omega$ satisfying

$$
\Omega-\bar{\omega}=-\frac{K}{4} \sum_{m=1}^{4} p_{m} \sin \left(\Omega \tau_{m}\right)
$$

For any delays $\tau_{m}$, the right hand side of Eq. (6) is, in general, an oscillatory function. Hence, with increasing stimulation strength $K$, the number of solutions $\Omega$ [Eq. (6)] increases, too. This leads to a multistability, which is undesirable for effective control. But with a proper choice of delays $\left(\tau_{1}=\tau_{3}, \tau_{2}=\tau_{4}\right)$ and polarities $\left(p_{1}=p_{2}=1, p_{3}=p_{4}=-1\right)$ we prevent the system from multistability and force it into a unique solution of Eq. (6) with $\Omega=\bar{\omega}$ for all $K$. From Eq. (5) we then get $\cos \phi_{m}=p_{m} \cos \left(\bar{\omega} \tau_{m}\right), \sin \phi_{m}=-p_{m} \sin \left(\bar{\omega} \tau_{m}\right)$, which provides delays appropriate for an equally distributed four-cluster state. We assess the amount of synchronization or clustering in Eq. (3) with the order parameters

$$
R_{n}(t) e^{i \Theta_{n}(t)}=\frac{1}{\mu} \int_{D} e^{i n \psi(\xi, t)} d \xi, \quad n=1, \ldots, 4
$$

defined in analogy to Eq. (4). Then conditions $R_{1}=R_{2}=R_{3}$ $=0$ and $R_{4}=1$, together with the above results imply $\bar{\omega}\left(\tau_{2}\right.$ $\left.-\tau_{1}\right)=\pi / 2+\pi k, k \in \mathbb{Z}$. From a practical point of view, delays close to zero and large delays might be not desirable. Very small delays might be difficult to realize in a practical application because of limiting minimal delays induced by the signal processing, on the other hand, extremely large delays might destroy the relation between the neuronal activity and the corresponding stimulation signal. Thus the delays from Fig. 2 are the most reasonable choice for our applications.

Also for the more realistic situation with nonzero coupling we use the ansatz $\psi(x, t)=\Omega t+\phi_{m}$, with delays $\tau_{1}=\tau_{3}$, $\tau_{2}=\tau_{4}$ and polarities $p_{1}=p_{2}=1, p_{3}=p_{4}=-1$. For sufficiently small coupling, Eq. (5) has a unique stable phase-locked solution $\psi(x, t)=\bar{\omega} t+\phi_{m}$ with (a)
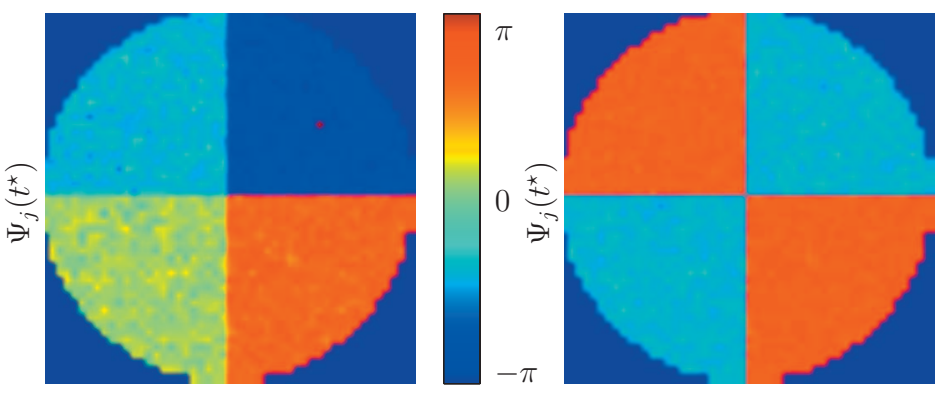

(b)

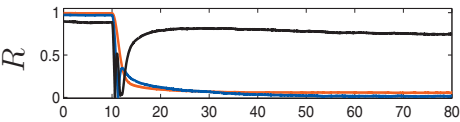

(c)

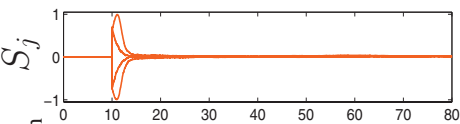

(d)
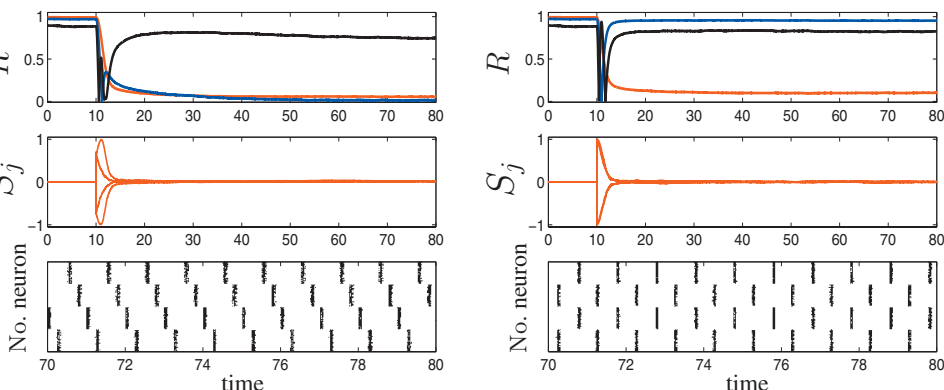

066209-3
FIG. 2. (Color) Stimulation-induced patterns of synchrony. Segmental stimulation with (i) delays $\tau_{1}=\tau_{3}=\frac{11}{8} T, \tau_{2}=\tau_{4}=\frac{9}{8} T$ and polarities $p_{1}$ $=p_{2}=1, p_{3}=p_{4}=-1$ (left) or (ii) $\tau_{1-4}=\frac{5}{4} T$ and $p_{1}$ $=p_{3}=1, p_{2}=p_{4}=-1$ (right) results in a rotatingwave-type four-cluster state (left) or a standingwave-type two-cluster state (right). (a) Snapshot of color coded phases of the individual oscillators at time 80. Time course of (b) order parameters $R_{1}$ (red line), $R_{2}$ (blue line), and $R_{4}$ (black line), and (c) stimulation amplitude $S_{j}$ of four representative neurons, each located in different segments. (d) Raster plot of the firing activity of all oscillators, where dots indicate neuronal firing (i.e., corresponding phases vanish $\bmod 2 \pi$ ). Parameters: $N=1976, C=0.1, K=1$ for $t \in[10,80]$, $K=0$ else, $A=0.002, T=1, a=1,\left\langle\omega_{j}\right\rangle=2 \pi,\left\{\omega_{j}\right\}$ Gaussian distributed with standard deviation $2 \pi / 1000$. In this paper the stochastic differential equation (2) is integrated with Euler's technique (time step of 0.005). 

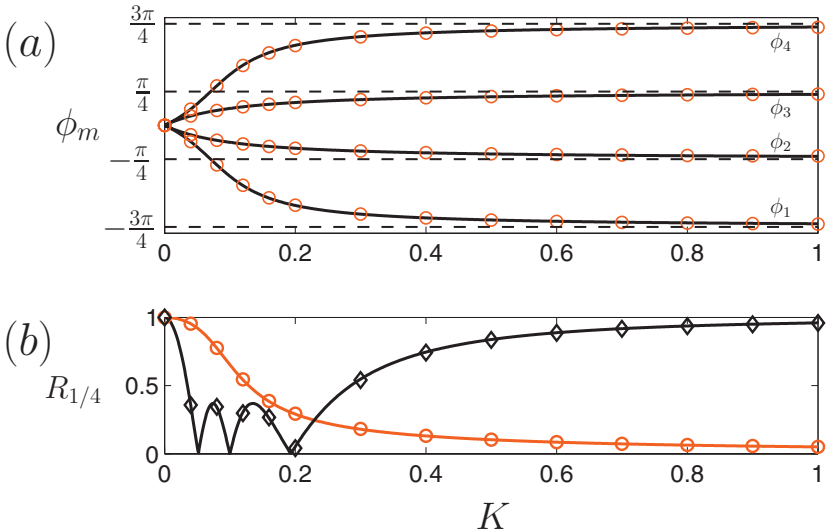

FIG. 3. (Color) Emergence of four-cluster states. (a) The phases $\phi_{1}, \ldots, \phi_{4}$ of the oscillators within the four clusters are plotted as a function of $K$. Numerical results of Eq. (2) (red circles) are compared with analytical results (see text, solid lines). (b) Corresponding order parameters [Eq. (7)]: analytically (red line) and numerically (red circles) determined $R_{1}$, analytical (black line) and simulated (black diamonds) $R_{4}$. Parameters: $N=716, C=0.1, A=0$, $\omega_{j}=2 \pi$.

$$
\begin{gathered}
\phi_{1}=-\phi_{4}= \begin{cases}-\arctan \frac{\varepsilon}{\sqrt{2}-\varepsilon} & \text { if } \varepsilon<\sqrt{2}, \\
-\pi / 2 & \text { if } \varepsilon=\sqrt{2}, \\
-\pi-\arctan \frac{\varepsilon}{\sqrt{2}-\varepsilon} & \text { if } \varepsilon>\sqrt{2},\end{cases} \\
\phi_{2}=-\phi_{3}=-\arctan \frac{\varepsilon}{\sqrt{2}+\varepsilon},
\end{gathered}
$$

where $\varepsilon=K / C$.

These analytical results show that due to multisite delayed feedback the synchronized state disappears, and a fourcluster state emerges. For increasing $K$ the phases $\phi_{1-4}$ approach an equally distributed four-cluster state as confirmed by numerical simulations of Eq. (2) [Fig. 3(a)]. Furthermore, in accordance with the numerical simulation, the order parameters $R_{1}$ and $R_{4}$ approach 0 and 1, respectively, as stimulation becomes strong enough $(K \gg C)$ [Fig. 3(b)].

The obtained analytical results, which are derived for a continuous system of identical oscillators, agree with the numerical results obtained in a system with equal natural frequencies. We also performed numerical simulations with wider frequency distributions, e.g., $\left\langle\omega_{j}\right\rangle=2 \pi / 5$ (standard deviation 0.1 , without figure). Multisite delayed feedback induces the desired spatiotemporal activity patterns also under such conditions and is robust with respect to variations of crucial parameters, e.g., $T \in\left[0,3 \frac{2 \pi}{\left\langle\omega_{j}\right\rangle}\right]$ and/or $K \in[-20,20]$. For a wide variation of $T$ one observes either a four-cluster state ( $T$ close to $\frac{2 \pi}{\left\langle\omega_{j}\right\rangle}$ or $3 \frac{2 \pi}{\left\langle\omega_{j}\right\rangle}$, Fig. 2, left) or a two-cluster state ( $T$ close to $0.5 \frac{2 \pi}{\left\langle\omega_{j}\right\rangle}$ or $1.5 \frac{2 \pi}{\left\langle\omega_{j}\right\rangle}$, similar to Fig. 2, right). For intermediate $T$ (covering also the nonresonant cases) a gradual transition between these states is observed. The above effects of the multisite-delayed feedback are preserved (a)
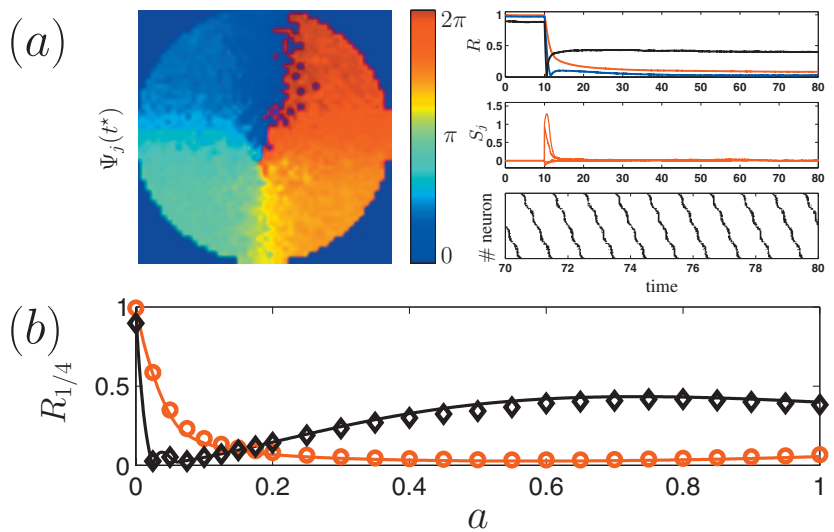

FIG. 4. (Color) Overlapping stimulation with sufficient electrode spacing causes smeared traveling-wave-type four-cluster states. (a) Phase distribution (left), order parameters $R_{1}$ (red), $R_{2}$ (blue), $R_{4}$ (black), stimulation amplitude $S_{j}$ of four representative neurons, and raster plot (right) for a simulation with inversely decaying stimulation for $a=1$. (b) Influence of the electrode distance on the resulting spatiotemporal phase pattern for an inversely decaying stimulation [analytic results: lines $\left(R_{1}\right.$ red, $R_{4}$ black), numerical simulations: symbols $\left(R_{1}\right.$ red circle, $R_{4}$ black diamond)]. $N=716, K=1, C=0.1, A=0.002$. Delays, polarities, and other parameters as in Fig. 2 (left).

in the case, where the stimulation profiles decay realistically (as modeled below), which underlines the robustness and applicability of the method.

\section{B. Overlapping stimulation}

Now, the effect of the stimulation decays inversely with increasing distance between neuron and electrode: $\rho_{j m}$ $=\left[l d \sqrt{1+4\left(\frac{d}{l}\right)^{2}}\right]^{-1}$, with $d=\left\|\mathbf{x}_{j}-\mathbf{X}^{m}\right\|$, where $\mathbf{X}^{m}$ denotes the position of electrode $m$, and $l=1 \mathrm{~mm}$ is the length of the contacts of a typical deep brain stimulation electrode $[29,30]$ [Fig. 4(a)]. Each network element is affected by a superposition of stimulation signals originating from all four electrodes. Consequently, the spatial distribution of the phases of the oscillators is smoother [Fig. 4(a)] compared to the case of strictly segmental stimulation [Fig. 2(a)]. The results of the noisy simulation and the predictions from the theoretical investigation coincide with each other, furthermore indicating the robustness of the investigated system with respect to the added noise [Fig. 4(b)]. The good agreement of the analytical results, derived for the case of identical oscillators without noise, and the numerical simulations indicates that the analytical results hold also for the inhomogeneous frequency distribution. For sufficient electrode spacing $(a>0.3)$ we obtain smeared rotating wave-type four-cluster states, which are characterized by intermediate values of $R_{4}$ and vanishing $R_{1}$ [Fig. 4(b)], corresponding to clusters which fire consecutively, but with a slight overlap [Fig. 4(a)]. In contrast, narrow electrode spacing $(0.15 \leqslant a \leqslant 0.2)$ causes a desynchronization $\left(R_{1,4}\right.$ close to zero) [Fig. 4(b)]. Also with overlapping stimulation the stimulation terms in Eq. (2) are drastically reduced as soon as the desired spatiotemporal dynamics is established [Fig. 4(a)]. 


\section{DISCUSSION}

Multisite delayed feedback stimulation provides an effective method for the control of spatiotemporal dynamics. Previously, elegant methods for the control of spatiotemporal dynamics, e.g., pinning control, have been developed [31]. The goal of such techniques was, e.g., to counteract chaos by stabilizing a predefined stationary or oscillatory state with instantaneous feedback signals [31]. In contrast, we use a delayed feedback approach, which enables us to evoke different spatiotemporal patterns of synchrony, depending on the selected arrangements of the delays. Applied to models with CPG-like architectures of interacting clusters of oscillators, multisite delayed feedback induces a coordinated sequential firing of the different clusters which might control different locomotor patterns (Fig. 2). Even in the unfavorable setting within a single homogenous population (see overlapping stimulation), our method causes (slightly smeared) CPG activity (Fig. 4). Multisite delayed feedback stimulation maintains spatially patterned synchrony with minimal amounts of stimulation. Hence multisite delayed feedback stimulation might be used to restore CPG activity in patients with incomplete spinal cord injury $[10,17]$ or gait ignition disorders [32].

\section{ACKNOWLEDGMENTS}

This study was supported by the Network of Excellence in Biosimulation (Grant No. 005137) and by IBM.
[1] Y. Kuramoto, Chemical Oscillations, Waves, and Turbulence (Springer, Berlin, 1984).

[2] S. Strogatz, Physica D 143, 1 (2000); G. Kozyreff, A. G. Vladimirov, and P. Mandel, Phys. Rev. Lett. 85, 3809 (2000); A. Pikovsky, M. Rosenblum, and J. Kurths, Synchronization, A Universal Concept in Nonlinear Sciences (Cambridge University Press, Cambridge, England, 2001); S. Boccaletti, J. Kurths, G. Osipov, D. L. Valladares, and C. Zhou, Phys. Rep. 366, 1 (2002); E. Mosekilde, Y. Maistrenko, and D. Postnov, Chaotic Synchronization: Applications to Living Systems (World Scientific, Singapore, 2002); D. M. Abrams and S. H. Strogatz, Phys. Rev. Lett. 93, 174102 (2004); H. Haken, Advanced Synergetics (Springer, Berlin, 1983); A. B. Neiman and D. F. Russell, Phys. Rev. Lett. 88, 138103 (2002); O. Sosnovtseva, D. Setsinsky, A. Fausboll, and E. Mosekilde, Phys. Rev. E 66, 041901 (2002).

[3] A. L. Benabid, P. Pollak, C. Gervason, D. Hoffmann, D. M. Gao, M. Hommel, J. E. Perret, and J. de Rougemont, Lancet 337, 403 (1991).

[4] P. A. Tass, Phase Resetting in Medicine and Biology (Springer, Berlin, 1999).

[5] M. G. Rosenblum and A. S. Pikovsky, Phys. Rev. Lett. 92, 114102 (2004a).

[6] O. V. Popovych, C. Hauptmann, and P. A. Tass, Phys. Rev. Lett. 94, 164102 (2005).

[7] C. Hauptmann, O. Popovych, and P. A. Tass, Biol. Cybern. 93, 463 (2005).

[8] P. A. Tass, C. Hauptmann, and O. Popovych, Int. J. Bifurcation Chaos Appl. Sci. Eng. 16, 1889 (2006).

[9] E. Marder and R. Calabrese, Physiol. Rev. 76, 687 (1996).

[10] V. Dietz, Clin. Neurophysiol. 114, 1379 (2003).

[11] J. Duysens and H. van de Crommert, Gait and Posture 7, 131 (1998).

[12] E. von Holst, Pfluegers Arch. Gesamte Physiol. Menschen Tiere 236, 149 (1935).

[13] M. Bellingham, Clin. Exp. Pharmacol. Physiol. 25, 847 (1998).

[14] R. Yuste, J. N. MacLean, J. Smith, and A. Lansner, Nat. Rev. Neurosci. 6, 477 (2005).

[15] A. H. Cohen, G. B. Ermentrout, T. Kiemel, N. Kopell, K. A. Sigvardt, and T. L. Williams, Trends Neurosci. 15, 434 (1992);
M. Golubitsky, I. Stewart, P. L. Buono, and J. J. Collins, Nature (London) 401, 693 (1999); C. M. A. Pinto and M. Golubitsky, J. Math. Biol. 53, 474 (2006).

[16] D. Johns, Semin Pediatr. Surg. 4, 152 (1995).

[17] V. Dietz and R. Müller, Brain 127, 2221 (2004).

[18] Note that our approach differs from standard functional electrical stimulation [M.R. Popovic, T. Keller, I. P. Pappas, V. Dietz, and M. Morari, IEEE Eng. Med. Biol. Mag. 20, 82 (2001)], where a neuroprosthesis generates short bursts of electrical pulses that when applied to muscles cause contractions.

[19] K. Pyragas, Phys. Lett. A 170, 421 (1992); 206, 323 (1995); W. Just, S. Popovich, A. Amann, N. Baba, and E. Scholl, Phys. Rev. E 67, 026222 (2003); N. B. Janson, A. G. Balanov, and E. Scholl, Phys. Rev. Lett. 93, 010601 (2004).

[20] M. Rosenblum and A. Pikovsky, Phys. Rev. E 70, 041904 (2004).

[21] O. Popovych, C. Hauptmann, and P. A. Tass, Biol. Cybern. 95, 69 (2006).

[22] P. A. Tass and H. Haken, Z. Phys. B: Condens. Matter 100, 303 (1996).

[23] D. Hansel, G. Mato, and C. Meunier, Europhys. Lett. 23, 367 (1993).

[24] In experiments instead of $\bar{W}(t)$, the activity of the whole CPG, one can also use $\bar{W}_{m}(t)$, the activity of the separate parts (i.e., subdomains $D_{m}$ ), and construct an integral activity of the whole CPG with the circular mean $\bar{W}(t)=\sum_{m=1}^{4} \bar{W}_{m}(t) / 4$, used for feedback [see Eq. (1)].

[25] We choose delays $\tau_{1}=\tau_{3}=\frac{11}{8} T, \tau_{2}=\tau_{4}=\frac{9}{8} T$, where $T=2 \pi / \bar{\omega}$, and polarities $p_{1}=p_{2}=1, p_{3}=p_{4}=-1$, where negative polarity adds a delay of $T / 2$.

[26] R. E. Mirollo and S. H. Strogatz, SIAM J. Appl. Math. 50, 1645 (1990).

[27] $R_{1}=1$ corresponds to perfect in-phase synchronization, and $R_{1}=0$ to absence of in-phase synchronization.

[28] We choose $\tau_{1-4}=5 / 4 T, p_{1}=p_{3}=1$, and $p_{2}=p_{4}=-1$.

[29] W. M. Grill and C. C. McIntyre, Thalamus Relat. Syst. 1, 269 (2001).

[30] K. Richardson, B. J. Gluckman, S. L. Weinstein, C. E. Glosch, J. B. Moon, R. P. Gwinn, K. Gale, and S. J. Schiff, Epilepsia 
44, 768 (2003).

[31] E. Ott, C. Grebogi, and J. A. Yorke, Phys. Rev. Lett. 64, 1196 (1990); E. R. Hunt, ibid. 67, 1953 (1991); H. Gang and H. Kaifen, ibid. 71, 3794 (1993); H. Gang and Qu Zhilin, ibid. 72, 68 (1994); M. Ding, E. J. Ding, W. L. Ditto, B. Gluckman,
V. In, J. H. Peng, M. L. Spano, and W. Yang, Chaos 7, 644 (1997); K. Pyragas, Philos. Trans. R. Soc. London, Ser. A 364, 2006 (2006).

[32] F. Rubino, Neurologist 8(4), 254 (2002). 